\title{
GlueVaR risk measures in capital allocation applications
}

\author{
Jaume Belles-Sampera Montserrat Guillén \\ Miguel Santolino
}

June 11, 2014

\begin{abstract}
Belles-Sampera et al. (2014) GlueVaR risk measures generalize the traditional quantile-based approach to risk measurement, while a subfamily of these risk measures has been shown to satisfy the tail-subadditivity property. In this paper we show how GlueVaR risk measures can be implemented to solve problems of proportional capital allocation. In addition, the classical capital allocation framework suggested by Dhaene et al. (2012) is generalized to allow the application of the Value-at-Risk (VaR) measure in combination with a stand-alone proportional allocation criterion (i.e., to accommodate the Haircut allocation principle). Two new proportional capital allocation principles based on GlueVaR risk measures are defined. An example based on insurance claims data is presented, in which allocation solutions with tail-subadditive risk measures are discussed.
\end{abstract}

Keywords: subadditivity, tails, distortion risk measure, capital allocation

\section{Introduction}

2 A risk measure provides information about the extreme, or tail, behavior of a 3 random variable associated with losses. In the fields of finance and insurance 4 their application determines the amount of capital to be held to guarantee a given 5 level of solvency. Capital allocation problems arise when a monetary amount 6 has to be distributed across different units. Typical examples of such problems 7 include the allocation of a sufficient amount of capital to cover the expected costs 8 of operational losses across departments, the total solvency capital requirement 
of a number of business lines and the total bonus pool to be shared among a company's employees, among others.

Guidelines as to how capital should be shared among a firm's units are determined in accordance with capital allocation principles, which are defined in terms of two components: (1) a capital allocation criterion and (2) a risk measure. The choice of the specific form that each component takes is essential insofar as different capital allocation solutions result from the combinations selected.

The Haircut allocation principle, for instance, combines a stand-alone proportional capital allocation criterion with the classical Value-at-Risk (VaR) measure; however, this principle was not originally included in the general theoretical framework provided by Dhaene et al. (2012) in which most of the capital allocation principles used in practice are accommodated. In this article we show how the Haircut allocation principle also fits in this framework.

In addition, we also examine the application of some recently introduced risk measures to the context of capital allocation problems. GlueVaR risk measures, which were initially defined by Belles-Sampera et al. (2014), can be expressed as a combination of VaR and Tail Value-at-Risk (TVaR) measures at different probability levels. These authors examined the properties of these new measures in the tails and showed that a subfamily of the GlueVaR family of risk measures satisfies the tail-subadditivity property, which means that the benefits of diversification can be preserved, at least in adverse scenarios.

Two new proportional capital allocation principles based on GlueVaR risk measures are proposed in this article. A discussion follows on how allocation principles based on GlueVaR measures are applied in practice and the implications of tail-subadditivity are described.

The article is structured as follows. The main concepts related to risk measures are briefly described in Section 2 and GlueVaR risk measures are introduced. Section 3 is devoted to the Haircut principle. In Section 4, GlueVaR risk measures are applied to capital allocation processes and two new proportional capital allocation principles based on GlueVaR risk measures are defined. An illustration of capital allocation solutions is provided in Section 5 and some concluding remarks are given. 


\section{Risk assessment using GlueVaR measures}

\subsection{Distortion risk measures}

A risk measure $\rho$ is a mapping from the set of random variables $X$ to the real line $\mathbb{R}, X \mapsto \rho(X) \in \mathbb{R}$. A class of risk measures extensively used in finance and insurance applications because of their appealing properties are the distortion risk measures. First introduced by Wang (Wang, 1995, 1996), a distortion risk measure is associated with distortion function $g$, where $g:[0,1] \rightarrow[0,1]$ is a function such that $g(0)=0, g(1)=1$ and $g$ is non-decreasing.

Consider a random variable $X$ and its survival function $S_{X}(x)=P(X>x)$. Function $\rho_{g}$ defined by $\rho_{g}(X)=\int_{-\infty}^{0}\left[g\left(S_{X}(x)\right)-1\right] d x+\int_{0}^{+\infty} g\left(S_{X}(x)\right) d x$ is known as a distortion risk measure where $g$ is the associated distortion function. Note that the convergence of the integrals used to define $\rho_{g}$ is not guaranteed for any $g$ and any $X$. Lack of convergence must be interpreted in the following way: random variable $X$ is too risky from the point of view of the risk assessor that uses $\rho_{g}$ as his risk measurement tool.

The VaR and TVaR measures can both be expressed as distortion risk measures. VaR at level $\alpha$ is the $\alpha$-quantile of the random variable $X$, i.e. $\operatorname{VaR}_{\alpha}(X)=$ $\inf \left\{x \mid F_{X}(x) \geq \alpha\right\}=F_{X}^{-1}(\alpha)$, where $F_{X}$ is the cumulative distribution function of $X$ and $\alpha$ is the confidence level $0 \leq \alpha \leq 1$. The associated distortion function of the VaR measure is,

$$
\psi_{\alpha}(u)=\left\{\begin{array}{lll}
0 & \text { if } \quad 0 \leq u<1-\alpha \\
1 & \text { if } \quad 1-\alpha \leq u \leq 1
\end{array}\right.
$$

TVaR at level $\alpha$ is defined as $\operatorname{TVaR}_{\alpha}(X)=\frac{1}{1-\alpha} \int_{\alpha}^{1} \operatorname{VaR} \lambda(X) d \lambda$. For continuous random variables, the TVaR measure is the mathematical expectation of losses given that these losses are greater than the associated VaR value. The distortion function for the TVaR is,

$$
\gamma_{\alpha}(u)=\left\{\begin{array}{lll}
\frac{u}{1-\alpha} & \text { if } & 0 \leq u<1-\alpha \\
1 & \text { if } & 1-\alpha \leq u \leq 1
\end{array} .\right.
$$

Distortion risk measures satisfy a set of properties including positive homo- 
geneity, translation invariance and monotonicity! When the associated distortion function is concave, the distortion risk measure is also subadditive (Denneberg, 1994, Wang and Dhaene, 1998, Wirch and Hardy, 2002). Unlike TVaR, VaR is not a subadditive risk measure (see, for instance, Denuit et al., 2005).

\subsection{GlueVaR risk measures}

GlueVaR risk measures have been defined by Belles-Sampera et al. (2014) within the class of distortion risk measures. Given a confidence level $\alpha, \alpha \in[0,1]$, the distortion function associated with a GlueVaR risk measure is:

$$
\kappa_{\beta, \alpha}^{h_{1}, h_{2}}(u)= \begin{cases}\frac{h_{1}}{1-\beta} u & \text { if } \quad 0 \leq u<1-\beta \\ h_{1}+\frac{h_{2}-h_{1}}{\beta-\alpha}[u-(1-\beta)] & \text { if } 1-\beta \leq u<1-\alpha \\ 1 & \text { if } \quad 1-\alpha \leq u \leq 1,\end{cases}
$$

where parameter $\beta$ is an extra confidence level such that $0 \leq \alpha \leq \beta \leq 1$. The shape of $\kappa_{\beta, \alpha}^{h_{1}, h_{2}}(u)$ is characterized by the two distorted survival probabilities $h_{1}$ and $h_{2}$ at levels $1-\beta$ and $1-\alpha$, respectively, where $0 \leq h_{1} \leq h_{2} \leq 1$. BellesSampera et al. (2014) showed that a GlueVaR risk measure can be expressed as a linear combination of standard risk measures. Figure 1 is a graphical representation of an example of the distortion function of a GlueVaR risk measure.

Lemma 1 Let $X$ be a random variable. Let $\alpha$ and $\beta$ be two probability levels such that $0 \leq \alpha \leq \beta \leq 1$, and let $h_{1}$ and $h_{2}$ be two survival probabilities such that $0 \leq h_{1} \leq h_{2} \leq 1$, then

$$
\operatorname{GlueVaR}_{\beta, \alpha}^{h_{1}, h_{2}}(X)=\omega_{1} \operatorname{TVaR}_{\beta}(X)+\omega_{2} \operatorname{TVaR}_{\alpha}(X)+\omega_{3} \operatorname{VaR}_{\alpha}(X),
$$

79 $\quad$ where $\omega_{1}=h_{1}-\frac{\left(h_{2}-h_{1}\right)(1-\beta)}{\beta-\alpha}, \omega_{2}=\frac{h_{2}-h_{1}}{\beta-\alpha}(1-\alpha)$ and $\omega_{3}=1-\omega_{1}-$ $\omega_{2}=1-h_{2}$.

Proof 1 The proof is straightforward and has been provided by Belles-Sampera et al. (2014).

${ }^{1}$ Additional properties for distortion risk measures can be found in Jiang (2008) and Balbás et al. (2009). 


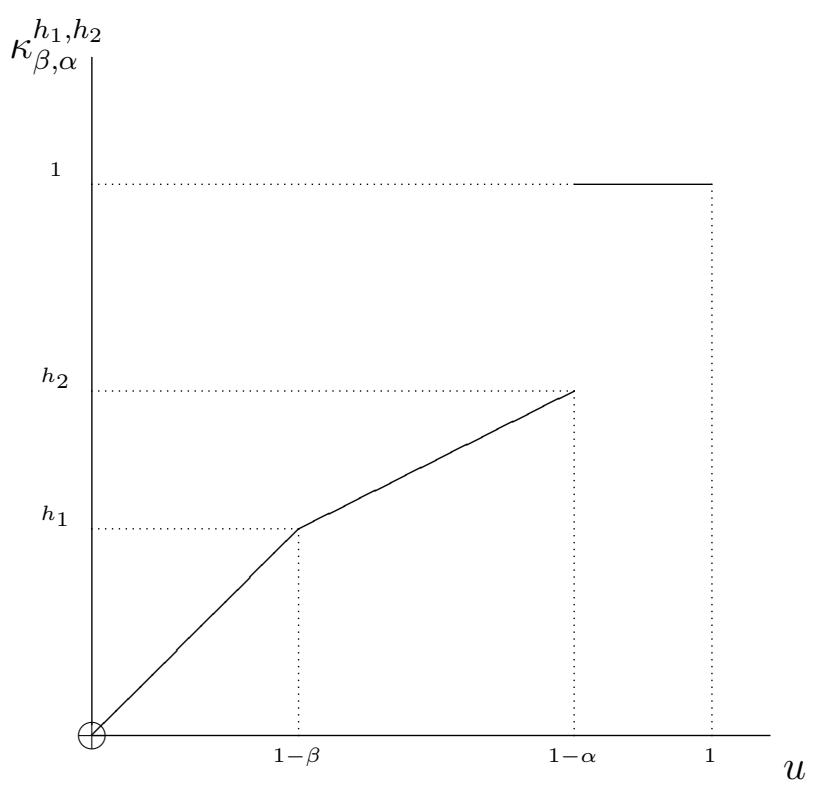

Figure 1: An example of GlueVar distortion function

As a consequence of Lemma 1, GlueVaR risk measures satisfy the subadditivity property if the weight associated with the VaR measure is null. BellesSampera et al. (2014) define the concept of tail-subadditivity. The idea is that the risk of a sum is smaller than or equal to the sum of risks only when focusing on the extreme region. Given a confidence level $\alpha$, the tail region of the random variable $Z$ is defined as $\mathcal{Q}_{\alpha, Z}:=\left\{\omega \mid Z(\omega)>s_{\alpha}(Z)\right\}$ where $s_{\alpha}(Z)$ is the $\alpha$-quantile $s_{\alpha}(Z)=\inf \left\{z \mid S_{Z}(z) \leq 1-\alpha\right\}$. Let $X, Y$ be two risks defined on the same probability space. The common tail for both risks is defined as $\mathcal{Q}_{\alpha, X, Y}:=\mathcal{Q}_{\alpha, X} \cap \mathcal{Q}_{\alpha, Y} \cap \mathcal{Q}_{\alpha, X+Y}$. This common tail is a key element to better understand the scope of the $\alpha$ tail-subadditivity, because it is the subset of the probability space where the subadditivity of the risk measure can be assured. Regarding the illustration provided in section 5, this is the common 5\%-right tail referred to in Table 1 Belles-Sampera et al. (2014) show that a GlueVaR risk measure is tail-subadditive if its associated with a distortion function that is concave in $[0,1-\alpha)$. 


\section{Risk capital allocation following the Haircut prin- ciple}

An extensive literature can be found discussing solutions to capital allocation problems (see, among others. Denault, 2001, Kalkbrener, 2005, Tsanakas, 2009, Buch et al., 2011, van Gulick et al., 2012). Some recent literature focuses on specific probability distributions of losses (Cossette et al., 2012, 2013), risk dependence structures (Cai and Wei, 2014), asymptotics of capital allocations based on commonly used risk measures (Asimit et al., 2011) or modifications of the optimization function to overcome limitations of allocations based on minimizing the loss function (Xu and Mao, 2013, Xu and Hu, 2012).

In this section we consider the framework suggested by Dhaene et al. (2012). This is a unifying framework in which a capital allocation problem is represented by means of three elements: a non-negative function (usually a norm), a set of weights, and a set of auxiliary random variables. However, the Haircut allocation principle could not be fitted into this framework, despite it being the most commonly used allocation criterion in practice (thanks to its simplicity).

Here, we propose a slight modification of the framework forwarded by Dhaene et al. (2012) by relaxing some of the conditions so as to include the Haircut capital allocation principle.

Assume that a capital $K>0$ has to be allocated across $n$ business units denoted by $i=1, \ldots, n$. Following Dhaene et al. (2012), any capital allocation problem can be described as the optimization problem given by

$$
\min _{K_{1}, K_{2}, \ldots, K_{n}} \sum_{j=1}^{n} v_{j} \mathbb{E}\left[\zeta_{j} D\left(\frac{X_{j}-K_{j}}{v_{j}}\right)\right] \quad \text { s.t. } \quad \sum_{j=1}^{n} K_{j}=K,
$$

with the following characterizing elements:

(a) a function $D: \mathbb{R} \rightarrow \mathbb{R}^{+}$;

(b) a set of positive weights $v_{i}, i=1, \ldots, n$, such that $\sum_{i=1}^{n} v_{i}=1$; and

(c) a set of random variables $\zeta_{i}, i=1, \ldots, n$, with $\mathbb{E}\left[\zeta_{i}\right]<+\infty$.

Unlike the original framework provided by Dhaene et al.(2012)), a distinction is made in (c) so that each $\zeta_{i}$ is now no longer forced to be positive with each $\mathbb{E}\left[\zeta_{i}\right]$ equal to 1 . Following this modification, the Haircut capital allocation solution can be obtained from the minimization problem (3). If a capital $K>0$ has to be 
allocated across $n$ business units, the Haircut allocation principle states that the capital $K_{i}$ to be assigned to each business unit must be

$$
K_{i}=K \frac{F_{X_{i}}^{-1}(\alpha)}{\sum_{j=1}^{n} F_{X_{j}}^{-1}(\alpha)} \quad \forall i=1, \ldots, n,
$$

where $X_{i}$ is the random loss linked to the $i$ th-business unit, $F_{X_{i}}^{-1}$ is the inverse of the cumulative distribution function of $X_{i}$ and $\alpha \in(0,1)$ is a given confidence level.

Let us consider $d_{i}=\min \left\{d \geq 1|0<| M^{d}\left[X_{i}\right] \mid<+\infty\right\}$ for all $i=1, \ldots, n$, where $M^{d}\left[X_{i}\right]=\mathbb{E}\left[X_{i}^{d}\right]$ is the moment of order $d>0$ of random variable $X_{i}$. Note that $d_{i} \geq 1$ for each $i$ to face a feasible capital allocation problem. In other words, if a business unit presents a random loss with no finite moments, then the risk taken by that business unit is not insurable.

The approach for fitting the Haircut allocation principle in the framework linked to the optimization problem (3) can be summarized as follows: if a constant $r_{i}$ must be expressed as $r_{i}=\mathbb{E}\left[\zeta_{i} X_{i}\right]$, then using $\zeta_{i}=\left(X_{i}^{d_{i}-1} / M^{d_{i}}\left[X_{i}\right]\right) r_{i}$ the solution is reached because $\mathbb{E}\left[\zeta_{i} X_{i}\right]=\mathbb{E}\left[\left(X_{i}^{d_{i}} / M^{d_{i}}\left[X_{i}\right]\right)\right] r_{i}=r_{i}$. Although an elegant approach is provided, the interpretation of the transformation made by $\zeta_{i}$ on $X_{i}$ is not trivial. We recommend to follow this strategy when there is none available alternative involving an interpretable $\zeta_{i}$.

Proposition 1 Let us consider a confidence level $\alpha \in(0,1)$. Then the three characterizing elements required to represent the Haircut allocation principle in the general framework defined by 3 are:

(a) $D(x)=x^{2}$,

(b) $v_{i}=\frac{\mathbb{E}\left[\zeta_{i} X_{i}\right]}{\sum_{j=1}^{n} \mathbb{E}\left[\zeta_{j} X_{j}\right]}, i=1, \ldots, n$; and

(c) $\zeta_{i}=\frac{X_{i}^{d_{i}-1}}{M^{d_{i}}\left[X_{i}\right]} F_{X_{i}}^{-1}(\alpha), i=1, \ldots, n$. 
Proof of Proposition 1. In this setting it is straightforward to show that the solution $\left\{K_{1}, K_{2}, \ldots, K_{n}\right\}$ to the minimization problem (3) is the Haircut allocation solution expressed by (4). Dhaene et al. (2012) show that, if function $D$ is fixed to be the Euclidean norm $\left(D(x)=x^{2}\right)$, then any solution to (3) can be written as

$$
K_{i}=\mathbb{E}\left[\zeta_{i} X_{i}\right]+v_{i}\left(K-\sum_{j=1}^{n} \mathbb{E}\left[\zeta_{j} X_{j}\right]\right), \quad \text { for all } \quad i=1, \ldots, n .
$$

In this setting, $v_{i}=\mathbb{E}\left[\zeta_{i} X_{i}\right] / \sum_{j=1}^{n} \mathbb{E}\left[\zeta_{j} X_{j}\right]$ for each $i$, so

$$
K_{i}=\mathbb{E}\left[\zeta_{i} X_{i}\right]+K \frac{\mathbb{E}\left[\zeta_{i} X_{i}\right]}{\sum_{j=1}^{n} \mathbb{E}\left[\zeta_{j} X_{j}\right]}-\mathbb{E}\left[\zeta_{i} X_{i}\right]=K \frac{\mathbb{E}\left[\zeta_{i} X_{i}\right]}{\sum_{j=1}^{n} \mathbb{E}\left[\zeta_{j} X_{j}\right]}
$$

And, finally, for all $i$ it is true that $\mathbb{E}\left[\zeta_{i} X_{i}\right]=F_{X_{i}}^{-1}(\alpha)$ because of (c). Therefore, each $K_{i}$ in the solution $\left\{K_{1}, K_{2}, \ldots, K_{n}\right\}$ is given by

$$
K_{i}=K \frac{F_{X_{i}}^{-1}(\alpha)}{\sum_{j=1}^{n} F_{X_{j}}^{-1}(\alpha)}
$$

Some particular comments on $v_{i}$ weights and $\zeta_{i}$ auxiliary random variables are here exposed. These comments are related to expression (5), the general solution of the optimization problem (3) when the Euclidean norm is used as $D$ function in the reference framework. Capital allocation principles driven by (5) can be thought of as two step allocation procedures: in a first step, a particular amount $\left(k_{i}=\mathbb{E}\left[\zeta_{i} X_{i}\right]\right)$ is allocated to each business unit and, as the sum of all these amounts should not add up to $K$ (i.e., $\sum_{j=1}^{n} k_{j} \neq K$ ), in the second step the difference $d=K-\sum_{j=1}^{n} k_{j}$ is allocated to the business units considering weights $v_{i}$. From this perspective, $k_{i}$ capitals are expected values of $X_{i}$ losses restricted to particular events of interest and, therefore, $\zeta_{i}$ auxiliary random variables are used to select those events of interest for each business unit. On the other hand, $v_{i}$ weights are related to the second step of the procedure, indicating how the difference $d$ between $K$ and $\sum_{j=1}^{n} k_{j}$ must be shared among business units. For a deeper interpretation of $v_{i}$ weights and $\zeta_{i}$ auxiliary random variables in more general cases, the interested reader is referred to Dhaene et al. (2012). 


\section{Proportional risk capital allocation principles us- ing GlueVaR}

Most of the proportional allocation principles found in the literature can be described in the framework suggested by Dhaene et al. (2012), where the characteristic elements are the Euclidean norm, weights $v_{i}=\mathbb{E}\left[\zeta_{i} X_{i}\right] /\left(\sum_{j=1}^{n} \mathbb{E}\left[\zeta_{j} X_{j}\right]\right)$, and a set of appropriate $\zeta_{i}$, for all $i=1, \ldots, n$. Following the notation used by these authors, we deal with business unit driven proportional allocation principles when $\zeta_{i}$ depends on $X_{i}$. If $\zeta_{i}$ depends on $S=\sum_{i=1}^{n} X_{i}$ then we have aggregate portfolio driven proportional allocation principles. In the former case, the marginal risk contributions of business units to the overall risk of the portfolio are not taken into account; in the latter, they are.

Here, two new proportional capital allocation principles are proposed using GlueVaR risk measures. Both principles share the characterizing elements $D(x)=$ $x^{2}$ and $v_{i}=\mathbb{E}\left[\zeta_{i} X_{i}\right] /\left(\sum_{j=1}^{n} \mathbb{E}\left[\zeta_{j} X_{j}\right]\right)$, for all $i=1, \ldots, n$. They only differ in the set of random variables $\zeta_{i}, i=1, \ldots, n$, which we present below for the case of continuous random variables $X_{i}$.

\subsection{Business unit driven proportional allocation principles us- ing GlueVaR}

Given two confidence levels $\alpha$ and $\beta$ in $(0,1), \alpha \leq \beta$, and two distorted survival probabilities $h_{1}$ and $h_{2}$, if $\zeta_{i}$ is fixed as

$$
\begin{aligned}
\zeta_{i} & =\omega_{1} \frac{\mathbb{1}\left[X_{i} \geq F_{X_{i}}^{-1}(\beta)\right]}{1-\beta}+\omega_{2} \frac{\mathbb{1}\left[X_{i} \geq F_{X_{i}}^{-1}(\alpha)\right]}{1-\alpha} \\
& +\omega_{3} \frac{X_{i}^{d_{i}-1}}{M^{d_{i}}\left[X_{i}\right]} F_{X_{i}}^{-1}(\alpha), \quad \text { for all } i=1, \ldots, n,
\end{aligned}
$$

then the business unit driven proportional allocation principle using GlueVaR $\mathrm{R}_{\beta, \alpha}^{h_{1}, h_{2}}$ can be represented in the modified capital allocation framework. Components of 
the solution $\left\{K_{1}, K_{2}, \ldots, K_{n}\right\}$ are expressed as

$$
K_{i}=K \frac{\operatorname{GlueVaR}_{\beta, \alpha}^{h_{1}, h_{2}}\left(X_{i}\right)}{\sum_{j=1}^{n} \operatorname{GlueVaR}_{\beta, \alpha}^{h_{1}, h_{2}}\left(X_{j}\right)}, \quad \text { for all } i=1, \ldots, n \text {. }
$$

Note that two different approaches are used to define random variables $\zeta_{i}$ for this principle. In the case of the $\operatorname{TVaR}_{\alpha}\left(X_{i}\right)$ for a continuous random variable $X_{i}$, an interpretable $\zeta_{i}$ is available and used, $\zeta_{i}=\frac{\mathbb{1}\left[X_{i} \geq F_{X_{i}}^{-1}(\alpha)\right]}{1-\alpha}$. On the other side, for $\operatorname{VaR}_{\alpha}\left(X_{i}\right)$ it is difficult to find random variables different than $\zeta_{i}=\left(X_{i}^{d_{i}-1} / M^{d_{i}}\left[X_{i}\right]\right) F_{X_{i}}^{-1}(\alpha)$ with an easier interpretation of the transformation made by $\zeta_{i}$ on $X_{i}$.

\subsection{Aggregate portfolio driven proportional allocation princi- ples using GlueVaR}

Similarly, if there exists a confidence level $\alpha^{*} \in(0,1)$ such that $F_{S}^{-1}(\alpha)=$ $\sum_{j=1}^{n} F_{X_{j}}^{-1}\left(\alpha^{*}\right)$, the aggregate portfolio driven proportional allocation principle using GlueVaR $\mathrm{R}_{\beta, \alpha}^{h_{1}, h_{2}}$ can be fitted to the modified capital allocation framework. In this case, $\zeta_{i}$ has to be equal to

$$
\begin{aligned}
\zeta_{i} & =\omega_{1} \frac{\mathbb{1}\left[S \geq F_{S}^{-1}(\beta)\right]}{1-\beta}+\omega_{2} \frac{\mathbb{1}\left[S \geq F_{S}^{-1}(\alpha)\right]}{1-\alpha} \\
& +\omega_{3} \frac{X_{i}^{d_{i}-1}}{M^{d_{i}}\left[X_{i}\right]} F_{X_{i}}^{-1}\left(\alpha^{*}\right), \quad \text { for all } i=1, \ldots, n .
\end{aligned}
$$

Each component of the solution $\left\{K_{1}, K_{2}, \ldots, K_{n}\right\}$ is then obtained as

$$
K_{i}=K \frac{\omega_{1} \mathbb{E}\left[X_{i} \mid S \geq F_{S}^{-1}(\beta)\right]+\omega_{2} \mathbb{E}\left[X_{i} \mid S \geq F_{S}^{-1}(\alpha)\right]+\omega_{3} F_{X_{i}}^{-1}\left(\alpha^{*}\right)}{\operatorname{GlueVaR}_{\beta, \alpha}^{h_{1}, h_{2}}(S)}
$$

Alternatively, another approach can be considered. There exists a set of confidence levels $\alpha_{j} \in(0,1)$ for all $j=1, \ldots, n$ such that $F_{S}^{-1}(\alpha)=\sum_{j=1}^{n} F_{X_{j}}^{-1}\left(\alpha_{j}\right)$. Therefore, the aggregate portfolio driven proportional allocation principle using GlueVaR ${ }_{\beta, \alpha}^{h_{1}, h_{2}}$ can also be fitted to the modified capital allocation framework. In this case, $\zeta_{i}$ has to be equal to 


$$
\begin{aligned}
\zeta_{i} & =\omega_{1} \frac{\mathbb{1}\left[S \geq F_{S}^{-1}(\beta)\right]}{1-\beta}+\omega_{2} \frac{\mathbb{1}\left[S \geq F_{S}^{-1}(\alpha)\right]}{1-\alpha} \\
& +\omega_{3} \frac{X_{i}^{d_{i}-1}}{M^{d_{i}}\left[X_{i}\right]} F_{X_{i}}^{-1}\left(\alpha_{i}\right), \quad \text { for all } i=1, \ldots, n .
\end{aligned}
$$

Each component of the solution $\left\{K_{1}, K_{2}, \ldots, K_{n}\right\}$ is then obtained as

$$
K_{i}=K \frac{\omega_{1} \mathbb{E}\left[X_{i} \mid S \geq F_{S}^{-1}(\beta)\right]+\omega_{2} \mathbb{E}\left[X_{i} \mid S \geq F_{S}^{-1}(\alpha)\right]+\omega_{3} F_{X_{i}}^{-1}\left(\alpha_{i}\right)}{\operatorname{GlueVaR}_{\beta, \alpha}^{h_{1}, h_{2}}(S)} .
$$

\section{Example of insurance risk capital allocation using GlueVaR}

An insurance database of claim costs is used to illustrate the adoption of GlueVaR measures in the context of risk capital allocation applications and to discuss its practical implications. Data were provided by a major Spanish motor insurer and have been previously analyzed in Bolancé et al. (2008), Guillén et al. (2011) and Belles-Sampera et al. (2013). The sample consists of $n=518$ observations of the cost of individual claims involving property damages $\left(X_{1}\right)$, medical expenses $\left(X_{2}\right)$ and the sum of those costs $\left(X_{1}+X_{2}\right)$. Amounts are expressed in thousands of euros.

Table 1 presents the risk measures when considering the empirical distribution. Risk measure values for $X_{1}+X_{2}$ under the most frequently used parametric distributions can be found in Belles-Sampera et al. (2014). Three GlueVaR measures are shown in Table 1, corresponding to different risk attitudes. GlueVaR $\mathrm{R}_{99.5 \%, 95 \%}^{11 / 30,2 / 3}$ reflects a balanced attitude, weighting $\mathrm{TVaR}_{99.5 \%}, \mathrm{TVaR}_{95 \%}$ and $\mathrm{VaR}_{95 \%}$ equally. $\mathrm{GlueVaR}_{99.5 \%, 95 \%}^{0,1}$ corresponds to a scenario in which a zero weight is allocated to $\mathrm{VaR}_{95 \%}$, the $\mathrm{TVaR} \mathrm{R}_{95}$ is overweighted and the lowest feasible weight is allocated to $\mathrm{TVaR}_{99.5 \%}$. Finally, GlueVaR $\mathrm{R}_{99.5 \%, 95 \%}^{1 / 20,1 / 8}$ reflects a more conservative attitude than that represented by using $\mathrm{VaR}_{95 \%}$ on its own. Table 1 is divided into two blocks. In the first, risk was calculated for the whole data set and in the second, contributions to the risk shown in the first block coming only from the $5 \%$-common tail were computed. Recall the definition of the $\alpha$-common tail provided in section 2.2 thus, in this second block, only the observations that lie simultaneously to the right of the $95 \%$ quantile of $X_{1}, X_{2}$ and $X_{1}+X_{2}$ were considered. The last column presents the concentration index, which is the ratio of the risk of $X_{1}+X_{2}$ 
divided by the sum of the risk of $X_{1}$ plus the risk of $X_{2}$. A concentration index smaller than one indicates subadditivity and, hence, a diversification effect.

Table 1: Risk assessment of claim costs using GlueVaR risk measures

$\begin{array}{lllll}\mathrm{X}_{1} & \mathrm{X}_{2} & \mathrm{X}_{1}+\mathrm{X}_{2} & \text { Difference }^{(*)} & \begin{array}{l}\text { Concentration } \\ \text { index }\end{array}\end{array}$
(a)
(b)
(c)
(a)+(b)-(c)
$(c) /((a)+(b))$

Whole domain

\begin{tabular}{lrrrrr}
\hline VaR $_{95 \%}$ & 38.8 & 6.4 & 47.6 & -2.4 & 1.05 \\
TVaR $_{95 \%}$ & 112.5 & 18.4 & 125.5 & 5.4 & 0.96 \\
TVaR $_{99.5 \%}$ & 440.0 & 54.2 & 479.0 & 15.2 & 0.97 \\
GlueVaR $_{99.5 \%, 95 \%}^{11 / 30,2 / 3}$ & 197.1 & 26.3 & 217.4 & 6.0 & 0.97 \\
GlueVaR & 0.1 & \\
Gl.5\%,95\% & 76.1 & 14.4 & 86.2 & 4.3 & 0.95 \\
GlueVaR $_{99.5 \%, 95 \%}^{1 / 20,2 / 8}$ & 61.7 & 9.4 & 72.1 & -1.0 & 1.01
\end{tabular}

Common 5\%-right tail

\begin{tabular}{lrrrrr}
\hline VaR $_{95 \%}$ & 0.0 & 0.0 & 0.0 & 0.0 & - \\
TVaR $_{95 \%}$ & 75.3 & 12.5 & 76.8 & 11.0 & 0.88 \\
TVaR $_{99.5 \%}$ & 411.3 & 46.7 & 426.7 & 31.3 & 0.93 \\
GlueVaR $_{99.5 \%, 95 \%}^{11 / 30,3}$ & 162.2 & 19.7 & 167.8 & 14.1 & 0.92 \\
GlueVaR \\
$99.5 \%, 95 \%$ & 37.9 & 8.7 & 37.9 & 8.7 & 0.81 \\
GlueVaR $_{99.5 \%, 95 \%}^{1 / 20,95 \%}$ & 23.4 & 3.0 & 24.2 & 2.2 & 0.92 \\
\hline
\end{tabular}

(*) Benefit of diversification.

In this example, $\mathrm{VaR}_{95 \%}$ and one of the GlueVaR measures are not subadditive in the whole domain, because their associated distortion functions are not concave in the whole $[0,1]$ interval. However, GlueVaR $\mathrm{V}_{99.5 \%, 95 \%}^{11 / 30,2 / 3}$, GlueVaR ${ }_{99.5 \%, 95 \%}^{0,1}$ and GlueVaR $\mathrm{R}_{99.5 \%, 95 \%}^{1 / 20,1 / 8}$ satisfy tail-subadditivity at confidence level $\alpha=95 \%$. Note that the concentration indexes smaller than one reveal that all the measures are subadditive in the tail.

We next illustrate a capital allocation application where total capital has to be allocated between the two units of risk, $X_{1}$ and $X_{2}$. Table 2 shows particular allocation solutions for two proportional risk capital allocation principles. 
A similar behavior is observed for the three GlueVaR risk measures. The capital is allocated primarily to risk $X_{1}$ regardless of the allocation criterion. Note that the percentages of capital allocated to $X_{1}$ are higher when the aggregate portfolio driven allocation criterion is used and a confidence level $\alpha^{*}=95.37 \%$ is set such that $F_{S}^{-1}(95 \%)=F_{X_{1}}^{-1}(95.37 \%)+F_{X_{2}}^{-1}(95.37 \%)$. This is an expected result, because the right tail of $X_{1}$ is fatter than that of $X_{2}$.

Let us focus on capital allocation solutions involving the aggregate portfolio driven criterion in which confidence levels $\alpha_{j}, j=1,2$ are not forced to be equal across the risk units. A notable fall in the risk allocated to $X_{1}$ is observed if an aggregate portfolio driven criterion with no constant level $\alpha^{*}$ and $\mathrm{GlueVaR}_{99.5 \%, 95 \%}^{1 / 20,2 / 8}$ is chosen.

This result is obtained because the impact on the quantile of $X_{1}$ is the opposite of that on $X_{2}$ when $\alpha_{j}, j=1,2$, are estimated as $F_{S}^{-1}(95 \%)=F_{X_{1}}^{-1}\left(\alpha_{1}\right)+$ $F_{X_{2}}^{-1}\left(\alpha_{2}\right)$, where $\alpha_{1}=94.78 \%$ and $\alpha_{2}=97.49 \%$. This particular risk measure is not subadditive in the whole domain and is tail-subadditive for these data. In fact, the associated quantiles for individual variables are $\operatorname{VaR}_{94.78 \%}\left(X_{1}\right)$ and $\operatorname{VaR}_{97.49 \%}\left(X_{2}\right)$, so the risk contribution of $X_{1}$ is underweighted compared to the risk of $X_{2}$.

\section{Conclusions}

Managers face capital allocation problems in multiple scenarios (e.g., when distributing total costs, aggregating reserves or assigning bonuses). Here, we have developed two new proportional capital allocation principles based on the GlueVaR risk measures introduced by Belles-Sampera et al. (2014). We showed that these two capital allocation principles may be accommodated within the capital allocation framework suggested by Dhaene et al. (2012) and, moreover, this framework is generalized to include the Haircut allocation principle.

The illustration we provide is based on real insurance claims data. The example shows that GlueVaR risk measures can be employed for capital allocation applications using the two proportional capital allocation principles proposed in Section 4. No major differences are found in the capital allocation solutions, except for one GlueVaR risk measure that is subadditive in the tail, though not when the whole domain is considered and varying quantile levels are allowed for each risk source. A certain degree of caution is therefore recommended when the aggregate portfolio driven criterion involving different $\alpha$-quantiles is used, given that the results seem to be sensitive to the impact of the quantile level on individual 
Table 2: Proportional capital allocation solutions using GlueVaR for the claims cost data

\begin{tabular}{|c|c|c|}
\hline & $\begin{array}{l}\text { Proportion allo- } \\
\text { cated to } X_{1}\end{array}$ & $\begin{array}{l}\text { Proportion allo- } \\
\text { cated to } X_{2}\end{array}$ \\
\hline GlueVaR $_{99.5 \%, 95 \%}^{11 / 30,2 / 3}$ & $88.21 \%$ & $11.79 \%$ \\
\hline GlueVaR $_{99.5 \%, 95 \%}^{0,1}$ & $84.07 \%$ & $15.93 \%$ \\
\hline GlueVaR $_{99.5 \%, 95 \%}^{1 / 20,1 / 8}$ & $86.79 \%$ & $13.21 \%$ \\
\hline
\end{tabular}

Aggregate portfolio driven with constant ${ }^{(a)} \alpha^{*}$

\begin{tabular}{|c|c|c|}
\hline GlueVaR $_{99.5 \%, 95 \%}^{11 / 30,2 / 3}(a)$ & $90.75 \%$ & $9.25 \%$ \\
\hline GlueVaR $_{99.5 \%, 95 \%}^{0,1}(a)$ & $87.83 \%$ & $12.17 \%$ \\
\hline GlueVaR ${ }_{99.5 \%, 95 \%}^{1 / 20,1 / 8}(a)$ & $88.06 \%$ & $11.94 \%$ \\
\hline
\end{tabular}

Aggregate portfolio driven with non constant ${ }^{(b)} \alpha_{j}$

\begin{tabular}{|c|c|c|}
\hline GlueVaR $_{99,5 \%, 95 \%}^{11 / 30,2 / 3}(b)$ & $89.93 \%$ & $10.07 \%$ \\
\hline GlueVaR $_{99.5 \%, 95 \%}^{0,1}{ }^{(b)}$ & $87.83 \%$ & $12.17 \%$ \\
\hline GlueVaR $_{99.5 \%, 95 \%}^{1 / 20,1 / 8}(b)$ & $81.55 \%$ & $18.45 \%$ \\
\hline \multicolumn{3}{|c|}{$\begin{array}{l}{ }^{(a)} \mathrm{A} \text { confidence level } \alpha^{*} \text { such that } F_{S}^{-1}(95 \%)=F_{X_{1}}^{-1}\left(\alpha^{*}\right)+F_{X_{2}}^{-1}\left(\alpha^{*}\right) \text {. In this case } \\
\alpha^{*}=95.37 \% \text {. }\end{array}$} \\
\hline \multicolumn{3}{|c|}{$\begin{array}{l}\text { (b) Confidence levels } \alpha_{j} \in(0,1) \text { are selected to satisfy } F_{S}^{-1}(95 \%)=F_{X_{1}}^{-1}\left(\alpha_{1}\right)+ \\
F_{X_{2}}^{-1}\left(\alpha_{2}\right) \text {. In this case } \alpha_{1}=94.78 \% \text { and } \alpha_{2}=97.49 \%\end{array}$} \\
\hline
\end{tabular}


risk sources.

\section{Acknowledgment}

The authors thank the Spanish Ministry of Science for support ECO2012-35584. Montserrat Guillén thanks ICREA Academia. The authors acknowledge the valuable comments and suggestions from the referees and the managing editor and, specially, the $\mathrm{T}_{\mathrm{E}} \mathrm{X}$ code provided by one of the referees to draw Figure 1

\section{References}

Asimit, A., Furman, E., Tang, Q., and Vernic, R. (2011). Asymptotics for risk capital allocations based on Conditional Tail Expectation. Insurance: Mathematics and Economics, (49):310-324.

Balbás, A., Garrido, J., and Mayoral, S. (2009). Properties of distortion risk measures. Methodology and Computing in Applied Probability, 11(3, SI):385399.

Belles-Sampera, J., Guillén, M., and Santolino, M. (2013). The use of flexible quantile-based measures in risk assessment. IREA Working Papers 2013, University of Barcelona, Research Institute of Applied Economics.

Belles-Sampera, J., Guillén, M., and Santolino, M. (2014). Beyond Value-at-Risk: GlueVaR distortion risk measures. Risk Analysis, 34(1):121-134.

Bolancé, C., Guillén, M., Pelican, E., and Vernic, R. (2008). Skewed bivariate models and nonparametric estimation for the CTE risk measure. Insurance: Mathematics and Economics, 43(3):386-393.

Buch, A., Dorfleitner, G., and Wimmer, M. (2011). Risk capital allocation for RORAC optimization. Journal of Banking and Finance, 35(11):3001-3009.

Cai, J. and Wei, W. (2014). Some new notions of dependence with applications in optimal allocations problems. Insurance: Mathematics and Economics, (55):200-209. 
Cossette, H., Côté, M., Marceau, E., and Moutanabbir, K. (2013). Multivariate distribution defined with Farlie-Gumbel-Morgenstern copula and mixed Erland marginals: Aggregation and capital allocation. Insurance: Mathematics and Economics, (52):560-572.

Cossette, H., Mailhot, M., and Marceau, E. (2012). TVaR-based capital allocation for multivariate compound distributions with positive continuous claim amounts. Insurance: Mathematics and Economics, (50):247-256.

Denault, M. (2001). Coherent allocation of risk capital. Journal of Risk, 4(1):134.

Denneberg, D. (1994). Non-Additive Measure and Integral. Kluwer Academic Publishers, Dordrecht.

Denuit, M., Dhaene, J., Goovaerts, M., and Kaas, R. (2005). Actuarial Theory for Dependent Risks. Measures, Orders and Models. John Wiley \& Sons Ltd, Chichester.

Dhaene, J., Tsanakas, A., Valdez, E. A., and Vanduffel, S. (2012). Optimal Capital Allocation Principles. Journal of Risk and Insurance, 79(1):1-28.

Guillén, M., Prieto, F., and Sarabia, J. M. (2011). Modelling losses and locating the tail with the Pareto Positive Stable distribution. Insurance: Mathematics and Economics, 49(3):454-461.

Jiang, L. (2008). Convexity, translation invariance and subadditivity for gexpectations and related risk measures. Annals of Applied Probability, 18(1):245-258.

Kalkbrener, M. (2005). An axiomatic approach to capital allocation. Mathematical Finance, 15(3):425-437.

Tsanakas, A. (2009). To split or not to split: Capital allocation with convex risk measures. Insurance: Mathematics and Economics, 44(2):268-277.

van Gulick, G., De Waegenaere, A., and Norde, H. (2012). Excess based allocation of risk capital. Insurance: Mathematics and Economics, 50(1):26-42.

Wang, S. S. (1995). Insurance pricing and increased limits ratemaking by proportional hazard transforms. Insurance: Mathematics and Economics, 17(1):4354. 
Wang, S. S. (1996). Premium calculation by transforming the layer premium density. ASTIN Bulletin, 26(1):71-92.

Wang, S. S. and Dhaene, J. (1998). Comonotonicity, correlation order and premium principles. Insurance: Mathematics and Economics, 22(3):235-242.

Wirch, J. L. and Hardy, M. R. (2002). Distortion risk measures: Coherence and stochastic dominance. IME Conference, Lisbon.

Xu, M. and Hu, T. (2012). Stochastic comparisons of capital allocations with applications. Insurance: Mathematics and Economics, (50):293-298.

Xu, M. and Mao, T. (2013). Optimal capital allocation based on the Tail MeanVariance model. Insurance: Mathematics and Economics, (53):533-543. 